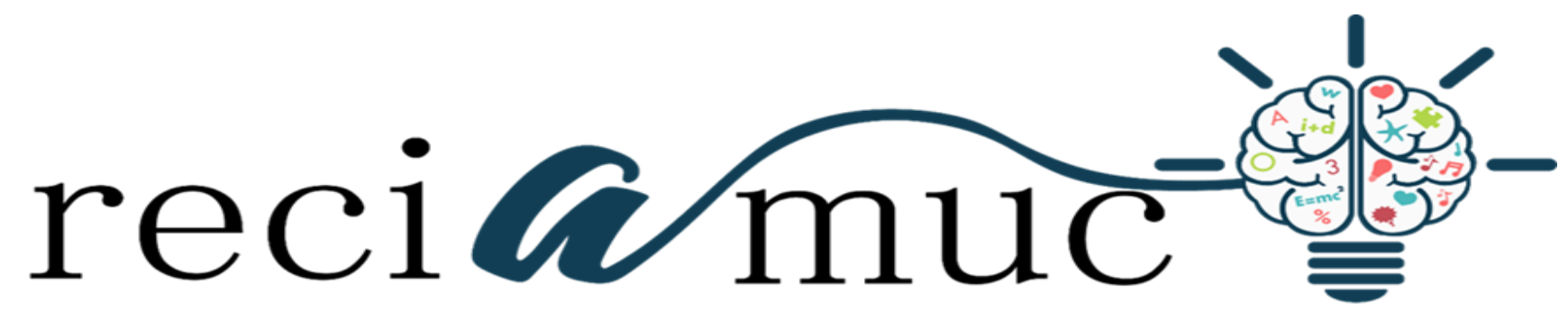

Revista cientifica de investigación actualización del mundo de las ciencias

Gabriela Elizabeth Martínez Ruiz a ; Josselin Estefania Villamarin Barreiro ${ }^{\text {b. }}$ Vivian

Dennise Palacios Vargas ${ }^{\text {c; }}$ Andrea Lisseth Oña Román ${ }^{\mathrm{d}}$

Cáncer de Mama: Factores de riesgo y respuesta

Breast Cancer: Risk factors and response

Revista Científica de Investigación actualización del mundo de las Ciencias. Vol. 3 núm., 3, julio, ISSN: 2588-0748, 2019, pp. 22-33

DOI: 10.26820/reciamuc/3.(3).julio.2019.22-33

URL: http://reciamuc.com/index.php/RECIAMUC/article/view/264

Código UNESCO: 3205 Medicina Interna

Tipo de Investigación: Artículo de Revisión

() RECIAMUC; Editorial Saberes del Conocimiento, 2019

Recibido: 28/04/2019

Aceptado: 19/05/2019

Publicado: 01/07/2019

Correspondencia: gemaral27@ gmail.com

a. Médico; Investigador Independiente; Guayaquil, Ecuador; gemaral27@gmail.com

b. Médico; Investigador Independiente; Guayaquil, Ecuador; josselinvillamrin@gmail.com

c. Médico; Investigador Independiente; Guayaquil, Ecuador; vivian-denisse@hotmail.com

d. Medico; Investigador Independiente; Guayaquil, Ecuador; andrea.or2989@gmail.com 


\section{Cáncer de Mama: Factores de riesgo y respuesta}

Vol. 3, núm. 3., (2019)

Gabriela Elizabeth Martínez Ruiz; Josselin Estefania Villamarin Barreiro; Vivian Dennise

Palacios Vargas; Andrea Lisseth Oña Román

\section{RESUMEN}

El cáncer es la segunda causa de muerte en todo el mundo. Se estima que, aproximadamente, una de cada seis muertes a nivel mundial son consecuencia de esta enfermedad. La presente investigación plasma los aspectos relacionados con los factores de riesgo asociados al cáncer de mama y la respuesta para la disminución de los factores modificables por medio de los planes de prevención internacionales. Para su desarrollo se llevó a cabo una recopilación y revisión de material documental bibliográfico. Existen factores de riesgos asociados al cáncer de mama que no se pueden modificar tales como la edad, antecedentes reproductivos y menstruales, tejido mamario denso, entre otros. Asimismo, existe un grupo de factores de riesgo modificables sobre los que se pueden aplicar medidas de protección y prevención de la enfermedad, tales como el consumo de alcohol, la alimentación, la lactancia materna y el uso prolongado de tratamiento hormonal. Las principales herramientas de prevención del cáncer de mama incluyen los programas de modificaciones o minimización de los factores de riesgo, los cuales con una eficaz aplicación pueden disminuir la incidencia del cáncer de mama en todo el mundo.

Palabras Claves: Cáncer; Mama; Factores; Riesgo; Respuesta. 


\title{
Cáncer de Mama: Factores de riesgo y respuesta
}

Vol. 3, núm. 3., (2019)

Gabriela Elizabeth Martínez Ruiz; Josselin Estefania Villamarin Barreiro; Vivian Dennise Palacios Vargas; Andrea Lisseth Oña Román

\begin{abstract}
Cancer is the second cause of death worldwide. It is estimated that approximately one out of every six deaths worldwide are a consequence of this disease. The present investigation captures the aspects related to risk factors associated with breast cancer and the response to the reduction of modifiable factors through international prevention plans. For its development a compilation and review of bibliographic documentary material was carried out. There are risk factors associated with breast cancer that can not be modified such as age, reproductive and menstrual history, dense breast tissue, among others. Likewise, there is a group of modifiable risk factors on which measures of protection and prevention of the disease can be applied, such as alcohol consumption, feeding, breastfeeding and the prolonged use of hormonal treatment. The main breast cancer prevention tools include programs to modify or minimize risk factors, which with an effective application can reduce the incidence of breast cancer worldwide.
\end{abstract}

Key Words: Cancer; Mom; Factors; Risk; Answer. 


\section{Cáncer de Mama: Factores de riesgo y respuesta}

Vol. 3, núm. 3., (2019)

Gabriela Elizabeth Martínez Ruiz; Josselin Estefania Villamarin Barreiro; Vivian Dennise

Palacios Vargas; Andrea Lisseth Oña Román

\section{Introducción.}

El cáncer de mama es aquel que comienza en los tejidos mamarios del cuerpo, puede ser de dos tipos principales: el carcinoma ductal, que como su nombre lo sugiere, comienza en los tubos que llevan leche desde la mama hasta el pezón y constituyen la mayoría de este tipo de cáncer, y el carcinoma lobular, el cual comienza en los lóbulos de las mamas. (Biblioteca Nacional de Medicina de los Estados Unidos, 2017)

A nivel general, el cáncer es la segunda causa de muerte en todo el mundo. En el año 2.015, fue responsable de 8,8 millones de defunciones. Aproximadamente, una de cada 6 muertes a nivel mundial son consecuencia de esta enfermedad. Alrededor de un $70 \%$ de las defunciones por cáncer ocurren en países de ingresos medios y bajos. "El impacto económico del cáncer es sustancial y va en aumento. Según las estimaciones, el costo total atribuible a la enfermedad en 2.010 ascendió a US\$ 1,16 billones”. (Organización Mundial de la Salud, 2018)

Más específicamente, el cáncer de mama se considera el tipo de cáncer más común en el mundo y la segunda causa de muerte por cáncer entre las mujeres de las Américas. Se estima que cada año se registran en esta región una cifra superior a los 462.000 casos nuevos, además de 100.000 defunciones por cáncer de mama. Los pronósticos apuntan que para el 2.030 se espera un aumento del cáncer de mama en las Américas, con un registro de 572.000 nuevos casos y alrededor de 130.000 defunciones. (Organización Panamericana de la Salud, 2018)

Existen factores de riesgo, que son hechos o circunstancias que pueden aumentar la probabilidad de que aparezca una enfermedad. Algunos de ellos son controlables, como beber alcohol, no obstante, otros tales como los antecedentes familiares, no se pueden controlar. Existen estudios que demuestran que cuantos más factores de riesgo se tenga, más aumenta el riesgo de aparecer la enfermedad, sin embargo, esto no quiere decir que infaliblemente el cáncer aparecerá. Por otra parte, existen algunas mujeres que presentan cáncer de mama sin presentar ningún factor de riesgo conocido, incluso no presentan antecedentes familiares. En conclusión, es importante entender los factores de riesgo ya que esto puede ayudar a conocer de qué manera prevenir el cáncer de mama. (Biblioteca Nacional de Medicina de los Estados Unidos, 2017) 


\section{Cáncer de Mama: Factores de riesgo y respuesta}

Vol. 3, núm. 3., (2019)

Gabriela Elizabeth Martínez Ruiz; Josselin Estefania Villamarin Barreiro; Vivian Dennise Palacios Vargas; Andrea Lisseth Oña Román

Para la Organización Panamericana de la Salud, (2015) la prevención en los programas de control del cáncer de mama juega un papel fundamental. "Las directrices sobre la prevención del cáncer de mama deben basarse en datos probatorios y actualizarse a medida que las investigaciones nuevas aporten información para la práctica clínica”. En base a esto, los expertos señalan, que el alcance del beneficio máximo con los programas preventivos, evitarían hasta un $50 \%$ de casos de cáncer de mama en todo el mundo. (p. 2,3)

De lo anterior se desprende la importancia del estudio de los factores de riesgos y la actualización de las investigaciones, con el propósito de adaptar las mejoras necesarias a los planes de prevención y erradicación de la enfermedad y sus consecuencias.

La presente investigación plasma los principales factores de riesgos asociados a la aparición del cáncer de mama y la respuesta general incluida actualmente a la planeación de estrategias preventivas internacionales.

\section{Materiales y Métodos.}

La presente investigación se realizó mediante una recopilación y revisión de material documental bibliográfico, usado para documentar y plasmar todo lo relacionado con los factores de riesgo asociados al cáncer de mama, así como la respuesta a los factores modificables incluida en los planes de prevención actuales a nivel internacional.

En tal sentido, se define la revisión documental como "el proceso dinámico que consiste esencialmente en la recogida, clasificación, recuperación y distribución de la información”. (A Latorre, D Rincón, J Arnal, 2003).

\section{Resultados.}

Factores de riesgo que no se pueden controlar.

Edad. "Su riesgo de cáncer de mama aumenta a medida que usted envejece. La mayoría de los cánceres se encuentra en mujeres de 55 años de edad en adelante". (Biblioteca Nacional de Medicina de los Estados Unidos, 2018) 


\section{Cáncer de Mama: Factores de riesgo y respuesta}

Vol. 3, núm. 3., (2019)

Gabriela Elizabeth Martínez Ruiz; Josselin Estefania Villamarin Barreiro; Vivian Dennise

Palacios Vargas; Andrea Lisseth Oña Román

Para Peña, González, Ávila, Utria, \& Más, (2017) “el riesgo de padecer cáncer se incrementa con la edad, en el caso del cáncer de mama, se incrementa a partir de la quinta década de la vida debido a la exposición previa y mantenida a numerosos factores de riesgo". (p. 287)

Mutaciones genéticas.

"Los cambios en los genes asociados con el cáncer de mama, como el BRCA1, BRCA2 y otros, aumentan su riesgo. Las mutaciones genéticas representan aproximadamente el 10\% de todos los casos de cáncer de mama”. (Biblioteca Nacional de Medicina de los Estados Unidos, 2018)

La Organización Panamericana de la Salud, (2015) menciona al respecto que existen factores genéticos que aumentan el riesgo de padecer cáncer, entre estos, cáncer de mama. Un 5\% al 10\%, aproximadamente, de los cánceres de mama se atribuyen a factores genéticos. Las personas portadoras de mutaciones en los genes BRCA1 o BRCA2, pueden presentar un riesgo calculado de que aparezca el cáncer de mama, alrededor de los 70 años de edad, con un aproximado de 55\% al $65 \%$, en el primer caso, y del $45 \%$ al $47 \%$, en el segundo caso. (p. 4)

\section{Tejido mamario denso.}

Un tejido mamario denso y menos tejido graso incrementa el riesgo de padecer cáncer de mama. Otro inconveniente de este tipo de tejido mamario es que dificulta que sea difícil ver tumores en un estudio mamográfico. (Biblioteca Nacional de Medicina de los Estados Unidos, 2018)

Antecedentes familiares o personales de cáncer de mama u otro cáncer.

Si existe un diagnóstico de cáncer de mama en la familia, la persona corre un mayor riesgo de padecer también de esa enfermedad. Si la persona ha tenido cáncer de mama, se encuentra en riesgo de que el cáncer de mama se vuelva a presentar. Otro factor que influye en la aparición de cáncer de mama son los antecedentes personales de cáncer de ovario. (Biblioteca Nacional de Medicina de los Estados Unidos, 2018)

Peña, González, Ávila, Utria, \& Más, (2017) citan a Figueroa, 2.002 y Peralta, 2.012, quienes obtuvieron en sus estudios, que el antecedente patológico familiar de cáncer de mama fue el factor predominante con una frecuencia de un 56,7\%. (p. 287) 


\section{Cáncer de Mama: Factores de riesgo y respuesta}

Vol. 3, núm. 3., (2019)

Gabriela Elizabeth Martínez Ruiz; Josselin Estefania Villamarin Barreiro; Vivian Dennise

Palacios Vargas; Andrea Lisseth Oña Román

\section{Antecedentes reproductivos y menstruales.}

“Tener su primer período antes de los 12 años de edad, comenzar la menopausia después de los 55 años, embarazarse después de los 30 años o no embarazarse jamás incrementan su riesgo". (Biblioteca Nacional de Medicina de los Estados Unidos, 2018)

Para Peña, González, Ávila, Utria, \& Más, (2017) "la menarquia temprana, desencadena una serie de descargas y trastornos hormonales..., ... con influencia en la carcinogénesis". Asimismo, se estima para la menopausia tardía un efecto carcinogénico hormonal similar. (Peña, González, Ávila, Utria, \& Más, 2017, p. 287)

Sifuentes, Castañeda, Lugo, \& Reyes, (2015) al respecto mencionan que la menopausia tardía, posterior a los 55 años de edad, aumenta en un 50\% el riesgo de cáncer mamario. Caso contrario, la menopausia temprana, sobre todo la quirúrgica, antes de los 40 años de edad, tiene un efecto protector en contra de este tipo de cáncer. "El embarazo a término en la mujer joven, especialmente antes de los 20 años, reduce de manera significativa el riesgo de cáncer mamario en la mayoría de los estudios revisados". (p. 664)

Raza.

"El cáncer de mama se diagnostica con mayor frecuencia a mujeres blancas que a mujeres afroamericanas/de raza negra, hispanas/latinas, asiáticas/de las Islas del Pacífico, o indias americanas/mujeres nativas de Alaska”. (Biblioteca Nacional de Medicina de los Estados Unidos, 2018)

Factores de riesgo que se pueden controlar.

\section{Radioterapia.}

Otro factor de riesgo del cáncer de mama lo constituye la exposición a radiaciones ionizantes, principalmente aquellas producidas por bombas atómicas, rayos $\mathrm{X}$ en las campanas diagnósticas de tuberculosis pulmonar, también por irradiación mamaria en el caso de mastitis puerperal, 


\section{Cáncer de Mama: Factores de riesgo y respuesta}

Vol. 3, núm. 3., (2019)

Gabriela Elizabeth Martínez Ruiz; Josselin Estefania Villamarin Barreiro; Vivian Dennise

Palacios Vargas; Andrea Lisseth Oña Román

irradiación por cáncer de mama, así como otras exposiciones producidas por el uso de la tecnología médica en la actualidad. (Sifuentes, Castañeda, Lugo, \& Reyes, 2015, p. 665)

\section{Consumo de alcohol.}

La Organización Mundial de la Salud, (2010) calcula que el uso nocivo del alcohol deja cada año unos 2,5 millones de muertes como consecuencia y que ocupa el tercer lugar entre los principales factores de riesgo para una mala salud en todo el mundo. "El consumo nocivo de alcohol es uno de los principales factores de riesgo evitables de los trastornos neuropsiquiátricos y otras enfermedades no transmisibles, como las cardiovasculares, la cirrosis hepática y diversos cánceres". (p. 3, 5)

Estudios de cohorte demuestran la asociación de la ingesta alcohólica con el cáncer de mama. El mayor consumo tiene repercusión en el aumento del riesgo, especialmente en la población de mujeres jóvenes. (Sifuentes, Castañeda, Lugo, \& Reyes, 2015, p. 665)

\section{Uso prolongado de terapia hormonal.}

"Tomar estrógeno y progestina combinados para la menopausia por 5 años o más incrementa su riesgo. No está claro si tomar pastillas anticonceptivas que contienen estrógeno aumenta su riesgo ni en qué medida”. (Biblioteca Nacional de Medicina de los Estados Unidos, 2018)

Por otro lado, el uso prolongado de tratamiento hormonal sustitutivo posterior a la menopausia se encuentra relacionado con un mayor riesgo de padecer cáncer de seno. "Un amplio ensayo aleatorizado, las mujeres que tomaron una combinación de estrógenos y progesterona por más de 5 años después de la menopausia presentaron un mayor riesgo de diagnóstico de cáncer de mama”. (Organización Panamericana de la Salud, 2015, p. 4)

\section{Peso e inactividad física.}

El sobrepeso u obesidad, después de la menopausia aumentan el riesgo de que aparezca el cáncer de mama con relación a aquellas personas que mantienen pesos saludables. Aquellas mujeres que 


\section{Cáncer de Mama: Factores de riesgo y respuesta}

Vol. 3, núm. 3., (2019)

Gabriela Elizabeth Martínez Ruiz; Josselin Estefania Villamarin Barreiro; Vivian Dennise Palacios Vargas; Andrea Lisseth Oña Román

no se ejercitan regularmente en toda su vida pueden aumentar el riesgo. (Biblioteca Nacional de Medicina de los Estados Unidos, 2018)

“La obesidad y la distribución de la grasa también se han estudiado como factor de riesgo de cáncer de mama". El ejercicio incrementa las concentraciones de estradiol, progesterona y aumenta la hormona folículo estimulante en la fase folicular. El ejercicio reduce la duración de la fase lútea y causa amenorrea secundaria, a largo plazo, especialmente en la adolescencia. (Sifuentes, Castañeda, Lugo, \& Reyes, 2015, p. 665)

Respuesta.

\section{Lactancia Materna (Factores Protectores).}

La lactancia materna es un factor protector. Diversos estudios han comprobado que amamantar parece reducir el riesgo de que aparezca el cáncer de mama. "Un examen de expertos reveló que por cada 12 meses que una mujer amamanta a su bebé, el riesgo relativo de cáncer de mama disminuye 4,3\%, y otro 7\% por cada bebé amamantado". (Organización Panamericana de la Salud, 2015 , p. 5)

\section{Actividad Física (Factores Protectores).}

El ejercicio es otro factor protector, realizarlo regularmente tiene un efecto protector contra el cáncer de mama. Estudios han revelado que se puede obtener una disminución promedio del riesgo del $25 \%$ al $40 \%$ entre mujeres físicamente activas en comparación con las menos activas. (Organización Panamericana de la Salud, 2015, p. 4)

De Los Santos \& Mariscal, (2018) refieren al respecto que existe una asociación significativa entre la actividad física y el desarrollo de cáncer de mama, por tal razón las recomendaciones de salud pública se deben enfocar en el fortalecimiento del mantenimiento de un peso saludable y de la actividad física, especialmente entre los jóvenes. Además de un factor de riesgo para el desarrollo de cáncer de mama, representa un factor de riesgo para múltiples enfermedades. (p. 43) 


\section{Cáncer de Mama: Factores de riesgo y respuesta}

Vol. 3, núm. 3., (2019)

Gabriela Elizabeth Martínez Ruiz; Josselin Estefania Villamarin Barreiro; Vivian Dennise

Palacios Vargas; Andrea Lisseth Oña Román

En el año 2.004 la Organización Mundial de la Salud, puso en marcha la "Estrategia mundial sobre régimen alimentario, actividad física y salud", vigente hasta la actualidad por cuanto para su desarrollo pleno y alcance se necesitarán esfuerzos combinados de muchas partes, durante varios decenios. Este Plan establece que la necesidad de combinar acciones racionales y eficaces desde lo más general a lo específico, esto incluye estrategias mundiales, regionales, nacionales y locales. Asimismo, es importante el seguimiento y evaluación de sus repercusiones. Uno de los principales objetivos de esta Estrategia Mundial es reducir los factores de riesgo de enfermedades crónicas relacionadas con una mala alimentación y con la falta de actividad física por medio de medidas de salud pública. (Organización Mundial de la Salud, 2019)

Con respecto al uso del nocivo del alcohol, es uno de los principales factores de riesgo contemplado en el Plan de acción de la estrategia mundial para la prevención y el control de las enfermedades no transmisibles de la Organización Mundial de la Salud. (Organización Mundial de la Salud, 2010, p. 26)

El mes de la Sensibilización sobre el Cancer de Mama, celebrado por primera vez en 1.985 en los Estados Unidos de América, ahora difundido al mundo entero, ha logrado una mayor conciencia sobre el impacto de este cáncer en las mujeres y en sus familias, la comunidad y la sociedad. Representa un catalizador para el aumento de los recursos a la investigación y el tratamiento en innumerables países. (Bustreo \& Chestnov, 2015)

\section{Conclusiones.}

Es fundamental estar informados y actualizados en cuanto a los factores de riesgo del cáncer de mama, de esta forma adoptar conductas de prevención de salud, que permitan evitar la enfermedad.

La prevención, consta en una buena parte, de la reducción de los factores de riesgo modificables, esta debe ser un componente fundamental de los programas en la lucha contra el cáncer de mama.

La prevención debe complementar los programas de detección temprana, diagnóstico y tratamientos oportuno, con la finalidad de reducir el número de muertes en todo el mundo por causa del cáncer de mama. 


\section{Cáncer de Mama: Factores de riesgo y respuesta}

Vol. 3, núm. 3., (2019)

Gabriela Elizabeth Martínez Ruiz; Josselin Estefania Villamarin Barreiro; Vivian Dennise

Palacios Vargas; Andrea Lisseth Oña Román

\section{Bibliografía.}

A Latorre, D Rincón, J Arnal. (2003). Bases Metodológicas de la Investigación Educativa. Barcelona: Ediciones Experiencia.

Biblioteca Nacional de Medicina de los Estados Unidos. (21 de Octubre de 2017). MedlinePlus. $\begin{array}{llllll}\text { Recuperado el } & 29 & \text { Mayo } & \text { de }\end{array}$ https://medlineplus.gov/spanish/ency/article/000913.htm

Biblioteca Nacional de Medicina de los Estados Unidos. (26 de Julio de 2018). MedlinePlus. $\begin{array}{llllll}\text { Recuperado el } & 27 & \text { Mayo } & \text { de }\end{array}$ https://medlineplus.gov/spanish/ency/patientinstructions/000830.htm

Bustreo, F., \& Chestnov, O. (30 de Octubre de 2015). Una cita importante: octubre es el Mes de Sensibilización sobre el Cáncer de Mama. Centro de Prensa de la Organización Mundial de la Salud, pág. 1. Recuperado el 29 de Mayo de 2019, de https://www.who.int/mediacentre/commentaries/breast-cancer-awareness/es/

De Los Santos, E., \& Mariscal, M. (Julio de 2018). EL CÁNCER DE MAMA EN NIÑAS Y ADOLESCENTES COMO POBLACIÓN VULNERABLE. Revista Cienytífica de Enfermeria(15), 36-49. Recuperado el 28 de Mayo de 2019, de https://rua.ua.es/dspace/bitstream/10045/77503/1/RECIEN_15_04.pdf

Organización Mundial de la Salud. (2010). Estrategia mundial para reducir el uso nocivo del alcohol. Departamento de Salud Mental y Abuso de Sustancias. Ginebra: OMS. $\begin{array}{llllll}\text { Recuperado } & \text { el } & 28 & \text { de } & \text { Mayo } & \text { de }\end{array}$ https://apps.who.int/iris/bitstream/handle/10665/44486/9789243599939_spa.pdf;jsessioni $\mathrm{d}=$ 24B0FC76C41CF4F3EC48411158287DA9? sequence $=1$

Organización Mundial de la Salud. (12 de Septiembre de 2018). who.int. Recuperado el 27 de Mayo de 2019, de https://www.who.int/es/news-room/fact-sheets/detail/cancer 


\section{Cáncer de Mama: Factores de riesgo y respuesta}

Vol. 3, núm. 3., (2019)

Gabriela Elizabeth Martínez Ruiz; Josselin Estefania Villamarin Barreiro; Vivian Dennise

Palacios Vargas; Andrea Lisseth Oña Román

Organización Mundial de la Salud. (2019). who.int. Recuperado el 28 de Mayo de 2019, de https://www.who.int/dietphysicalactivity/goals/es/

Organización Panamericana de la Salud. (2015). PREVENCIÓN: FACTORES DE RIESGO Y PREVENCIÓN DEL CÁNCER DE MAMA. Ginebra: OMS. Recuperado el 28 de Mayo de 2019, de https://www.paho.org/hq/dmdocuments/2015/prevencion-factores-riesgo.pdf

Organización Panamericana de la Salud. (2018). paho.org. Recuperado el 27 de Mayo de 2019, de https://www.paho.org/hq/index.php?option=com_docman\&view=download\&category_sl ug=estadisticas-mapas-4868\&alias $=46503$-epidemiologia-cancer-de-mama-en-lasamericas-2018\&Itemid $=270 \& l a n g=e n$

Peña, Y., González, M., Ávila, D., Utria, L., \& Más, Y. (13 de Diciembre de 2017). Factores de riesgo para padecer cáncer de mama en la población femenina. Revista Finlay, 13(4), 283289. Recuperado el 27 de Mayo de 2019, de http://scielo.sld.cu/pdf/rf/v7n4/rf08407.pdf

Ríos, R. (09 de 2017). www.eumed.net. (E. Grupo de investigación (SEJ 309) eumed.net de la Universidad de Málaga, Ed.) Obtenido de www.eumed.net: http://www.eumed.net/libros/libro.php?id=1662

Sifuentes, A., Castañeda, L., Lugo, M., \& Reyes, M. (Noviembre de 2015). Factores de riesgo asociados con el cáncer de mama en mujeres del estado de Durango México. Ginecología y Obstetricia de México, 83(15), 662-669. Recuperado el 27 de Mayo de 2019, de https://www.medigraphic.com/pdfs/ginobsmex/gom-2015/gom1511b.pdf

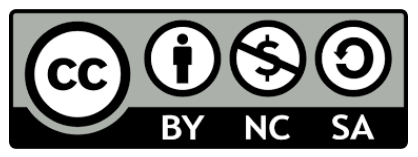

RECONOCIMIENTO-NOCOMERCIAL-COMPARTIRIGUAL

CC BY-NC-SA

ESTA LICENCIA PERMITE A OTROS ENTREMEZCLAR, AJUSTAR Y CONSTRUIR A PARTIR DE SU OBRA CON FINES NO

COMERCIALES, SIEMPRE Y CUANDO LE RECONOZCAN LA AUTORÍA Y SUS NUEVAS CREACIONES ESTÉN BAJO UNA LICENCIA CON LOS MISMOS TÉRMINOS. 